\section{Rosai-Dorfman disease as a differential diagnosis of cervical adenopathy}

\author{
Victor Giovannino Accetta ${ }^{1}$, Vinícius Campos Oliveira ${ }^{1}$, \\ Sílvia Migueis Picado Petrarolha², Angelo Sementilli3,1, Rogério Aparecido Dedivitis ${ }^{4,5}$
}

\begin{abstract}
Introduction: Rosai-Dorfman disease (RDD), also known as sinus histiocytosis with massive lymphadenopathy $(\mathrm{SHML})$, is a rare benign idiopathic disease that affects both nodal and extranodal sites. Case report: A female patient aged 27 years with right side cervical adenopathy of levels IV and V, both mobile, painless and of fibroelastic consistency, with no signs of inflammation was evaluated. Histopathology of the lymph node biopsy showed preserved lymphoid architecture and presence of numerous mature histiocytes compatible with RDD.
\end{abstract}

Keywords: histiocytosis; emperipolesis; hemophagocytic lymphohistiocytosis.

How to cite: Accetta VG, Oliveira VC, Petrarolha SMP, Sementilli A, Dedivitis RA. Rosai-Dorfman disease as a differential diagnosis of cervical adenopathy. Arch Head Neck Surg. 2020;49:e0010.2020. https://doi.org/10.4322/ahns.2020.0010

\section{Introduction}

Rosai-Dorfman disease (RDD), also known as sinus histiocytosis with massive lymphadenopathy (SHML), it is a rare condition that affects all age groups, including pediatric patients. It is a non-Langerhans cell histiocytosis most commonly presented as painless cervical lymphadenopathy, although extranodal manifestation has also been observed in at least one third of patients, with most evident involvement of the head and neck region'.

This disease is usually self-limiting, but can sometimes be life-threatening 1 . The factors that influence disease recurrence are still not well understood; however, hypotheses have been raised that this condition may result from changes in immune response and infections caused by certain viral and bacterial pathogens, such as Varicella Zoster Virus (VZV), Epstein-Barr Virus (EBV), Cytomegalovirus (CMV), Brucella, Klebsiella, among others².

\section{Case report}

A 27-year-old Caucasian female patient sought emergency care with a history of cervical mass for a month. She reported tiredness, hyporexia and vomiting, and had an axillar temperature of $39^{\circ} \mathrm{C}$. She also reported having 
lower limb pain, diffuse arthralgia, loss of appetite, and chills with night sweats for the past six months. Right side of the level IV cervical $(1.5 \mathrm{~cm})$ and supraclavicular $(1 \mathrm{~cm})$ lymph nodes were evidenced, both mobile, painless and of fibroelastic consistency, with no signs of inflammation. No lesions in the upper aerodigestive tract were observed at the locoregional examination. Laboratory tests showed no changes and serology was negative for mono-like diseases. No suspicious lesions were found at the locoregional examination. Cervical lymphadenectomy presented chronic lymphadenitis with intense sinus histiocytosis suggestive of RDD, with subsequent diagnostic confirmation by immunohistochemistry (IHC), which showed lymphoid architecture and presence of numerous mature histiocytes - Figures 1 to 3 . As the patient did not present any systemic manifestations after treatment with corticosteroids (Prednisone $30 \mathrm{mg} /$ day for 4 weeks), only surgical excision of the right supraclavicular lymph node was performed, without complications.

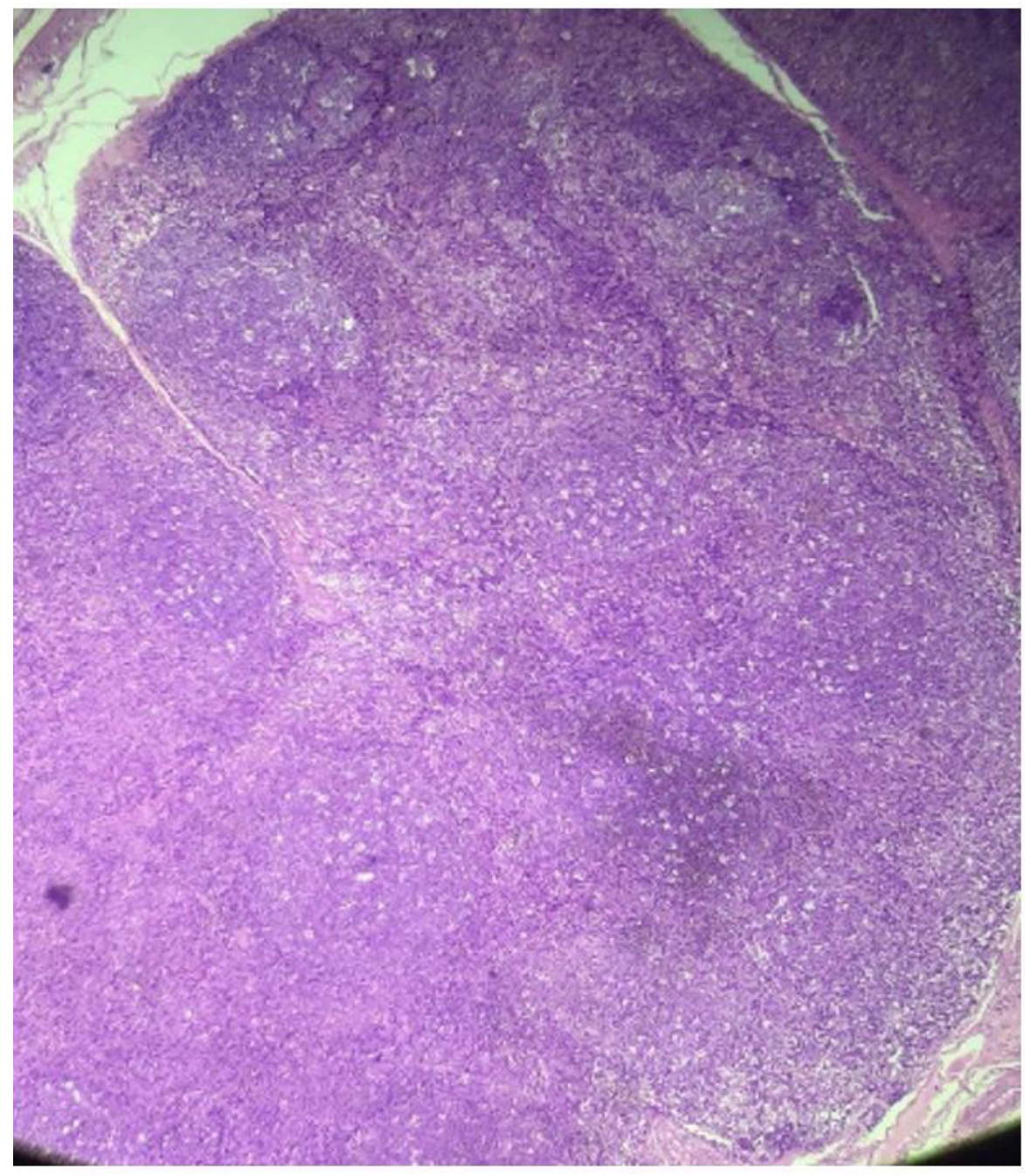

Figure 1. Photomicrography showing reactional lymphoid population with the presence of several immunoblasts (red circle), HE, 100x. 


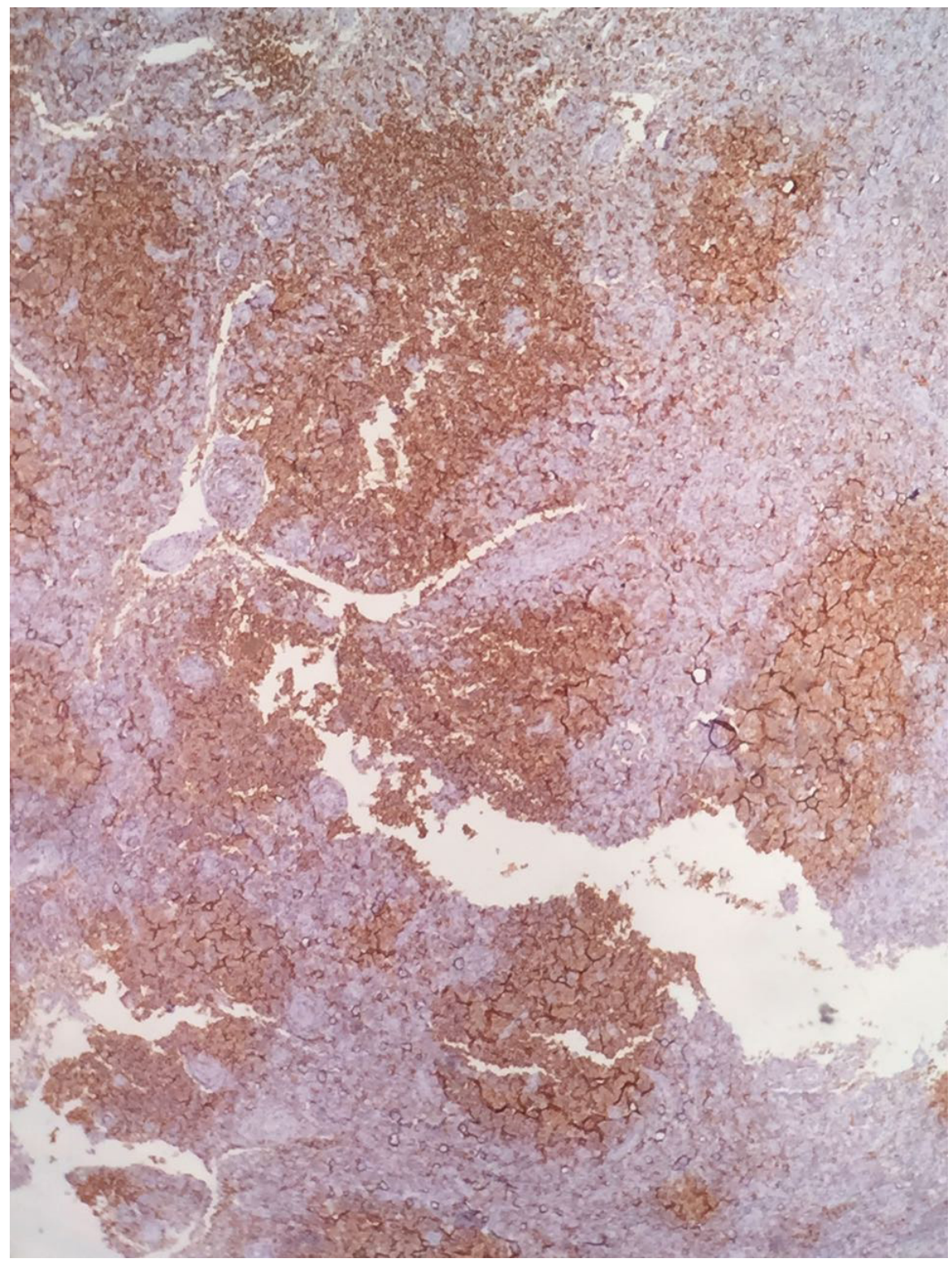

Figure 2. Photomicrography showing B lymphocytes in the germinative center; CD20+ immunohistochemistry. X100.

\section{Discussion}

Lymphadenectomy is rarely caused by RDD in children and young adults. Since RDD was first described in 1969, several cases of nodal and extranodal involvement have been reported ${ }^{2}$. RDD has two main forms of presentation: the first form affects the lymph nodes (SHML), with rare systemic manifestation in other organs; the second one involves only the skin, without any systemic or nodal disease even after long-term follow-up. These two forms are considered as different clinical entities².

Extranodal involvement has been observed in $25-43 \%$ of cases, whereas focal lymph node involvement has been found in $57 \%$ of cases. Painless 


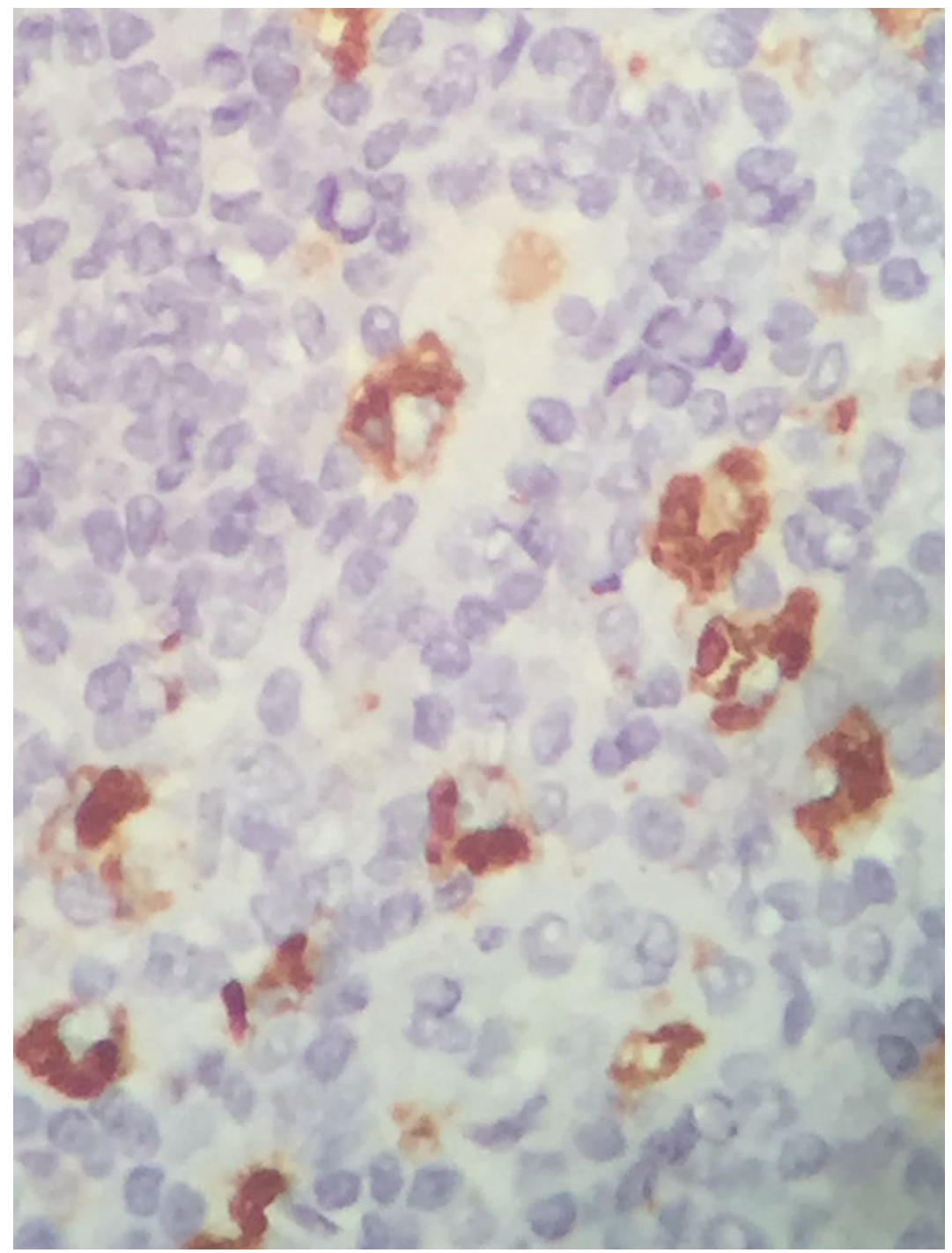

Figure 3. Photomicrography showing B macrophages with possible lymphocytes phagocytosis (emperipolesis); CD68+ immunohistochemistry, x400.

cervical lymphadenopathy is the most frequent initial symptom of this disease, reported by nearly $90 \%$ of patients ${ }^{2}$. In this patient, it was possible to palpate an enlarged lymph node from the cervical supraclavicular and internal jugular regions to the clavicle. Apparently, there is no relationship between the nodal disease and the extranodal sites, which can occur separately. The RDD etiology is uncertain, although infectious agents such as the EBV and VZV are important in its pathogenesis ${ }^{2}$. Typically, the disease occurs with insidious onset, prolonged active phase, and eventual spontaneous remission with subsequent recurrences ${ }^{3}$.

Imaging examinations can be useful in assessing the extent of RDD and histopathology is necessary to confirm its diagnosis. The presence of cytologic atypia and the aggressive clinical course of the disease establish the diagnosis in most cases $^{3}$. S-100 protein positivity a differential diagnostic aspect ${ }^{1}$. 


\section{*Correspondence}

Rogério Aparecido Dedivitis

Av. Conselheiro Nébias, $444,16^{\circ}$ andar CEP 11045-000, Santos (SP), Brasil

E-mail: dedivitis.hns@uol.com.br

\section{Authors information}

VGA - Medicine Student, Universidade Metropolitana de Santos. VCO Medicine Student, Universidade Metropolitana de Santos. SMPP Assistant, Department of Head and Neck Surgery, Hospital Ana Costa. AS - Chair, Department of Pathology, Universidade Federal de São Paulo. Professor Titular de Patologia da Universidade Metropolitana de Santos. RAD - Assistant Professor, Department of Surgery, University of São Paulo School of Medicine, Chair, Department of Scientific Methodology, Universidade Metropolitana de Santos.
Differential diagnosis includes several lymphoreticular malignancies such as lymphoma, Hodgkin's disease, histiocytic neoplasms and monocytic leukemia, which present similar histopathological characteristics.

Because SHML is considered an uncommon and self-limiting disease, there is no ideal protocol for its treatment. Half of the cases are resolved spontaneously. In patients with high fever alone, corticosteroid therapy may be indicated ${ }^{4}$. Surgical debulking is necessary in cases where lymph node enlargement induces important symptoms, such as when the airways are obstructed or vital organs are compressed. Radical surgery is not usually indicated considering the typically benign course of $\mathrm{RDD}^{5}$.

\section{References}

1. Lai KL, Abdullah V, Ng KS, Fung NS, van Hasselt CA. Rosai-Dorfman disease: Presentation, diagnosis, and treatment. Head Neck J Sci Spec. 2013;35(3):85-8. http://dx.doi.org/10.1002/hed.21930. PMid:22083607.

2. Kutlubay Z, Bairamov O, Sevim A, Demirkesen C, Mat MC. Rosai-Dorfman disease: A case report with nodal and cutaneous involvement and review of the literature. Am J Dermatopathol. 2014;36(4):353-7. http://dx.doi.org/10.1097/ DAD.0b013e31829e5564. PMid:23863552.

3. Mar WA, Yu JH, Knuttinen MG, Horowitz JM, David O, Wilbur A, Menias CO. Rosai-Dorfman disease: Manifestations outside of the head and neck. AJR Am J Roentgenol. 2017;208(4):721-32. http://dx.doi.org/10.2214/AJR.15.15504. PMid:28140608.

4. Padoveze EH, Alvarenga ML, Falzoni R, Rabay FMO. Doença de Rosai-Dorfman cutânea: relato de caso e revisão de literatura. Bras Patol Med Lab. 2011;47(1):65-9. http://dx.doi.org/10.1590/S1676-24442011000100009.

5. Pinto DCG, Vidigal TA, Castro B, Santos BH, Nicodemos JAS. Doença de Rosai-Dorfman como diagnóstico diferencial de linfadenopatia cervical. Rev Bras Otorrinolaringol. 2008;74(4):632-5. http://dx.doi.org/10.1590/ S0034-72992008000400025. 